ISSN 0103-9954

\title{
OCORRÊNCIA DE Hypsipyla ferrealis Hampson (LEPIDOPTERA: PYRALIDAE) EM ANDIROBA NO ESTADO DO ACRE
}

\author{
OCCURRENCE OF Hypsipyla ferrealis Hampson (LEPIDOPTERA: PYRALIDAE) IN ANDIROBA IN \\ THE STATE OF ACRE
}

Rodrigo Souza Santos ${ }^{1}$ André Schatz Pellicciotti ${ }^{2}$

\begin{abstract}
RESUMO
Relata-se a ocorrência de Hypsipyla ferrealis Hampson no Estado do Acre, infestando sementes de andirobeiras, oriundas do município de Senador Guiomard - AC. No Laboratório de Entomologia da Embrapa Acre, as sementes foram depositadas em bandejas plásticas contendo areia umedecida e acondicionadas no interior de uma gaiola telada. Diariamente, a gaiola foi inspecionada à procura de insetos emergidos, os quais foram identificados como Hypsipyla ferrealis, lepidóptero comumente associado infestando sementes de andirobeira. Este é o primeiro registro desta espécie ocorrendo em sementes de andirobeira no estado do Acre.
\end{abstract}

Palavras-chave: broca-da-semente da andiroba; Carapa guianensis; Meliaceae.

\begin{abstract}
The occurrence of Hypsipyla ferrealis Hampson was reported in the state of Acre, Brazil, infesting andiroba Retirar as palavras "the county of" da frase, pois municipality é sinônimo de county, ou seja, está em duplicidade no texto esta informação in plastic trays containing moistened sand and placed into a net cage at Embrapa Entomology Laboratory in Acre state. The cage was daily inspected in order to find emerged insects which were identified as Hypsipyla ferrealis, a lepidopteran commonly associated as an andiroba seed pest. This is the first record of this species occurring in andiroba seeds in Acre state.
\end{abstract}

Keywords: andiroba seed borer; Carapa guianensis; Meliaceae.

\section{INTRODUÇÃO}

A andirobeira pertence à família Meliaceae e ao gênero Carapa, sendo que na Amazônia brasileira existem duas espécies: Carapa guianensis Aublet (andiroba) e Carapa procera DeCandolle (andirobinha). No entanto, ambas são tratadas comercialmente como uma só espécie, e conhecidas pelo mesmo nome vulgar "andiroba" (FERRAZ et al., 2002), que significa, na língua indígena, sabor amargo (MENEZES, 2005).

Com relação à sua distribuição geográfica, Carapa guianensis ocorre no sul da América Central,
Colômbia, Venezuela, Suriname, Guiana Francesa, Brasil, Peru, Paraguaie nas ilhas do Caribe. Já Carapa procera é encontrada na África, nos Neotrópicos e ao norte da América do Sul, abrangendo o Brasil, o Suriname e a Guiana Francesa (FERRAZ et al., 2002). No Brasil, Carapa guianensis ocorre em toda bacia amazônica (FERRAZ et al., 2003), enquanto que Carapa procera está restrita a algumas regiões amazônicas (MENDONÇA e FERRAZ, 2007), não ocorrendo naturalmente em alguns estados, como o Acre, por exemplo (BOUFLEUER, 2004).

O óleo extraído das sementes de Carapa guianensis é um dos produtos florestais não

1 Biólogo, Dr., Pesquisador do Centro de Pesquisa Agroflorestal do Acre (Embrapa Acre), Rod. BR 364, Km 14, Caixa Postal 321, CEP 69900-970, Rio Branco (AC), Brasil. rodrigo.s.santos@embrapa.br

2 Engenheiro Florestal, Secretaria de Desenvolvimento Florestal, da Indústria, do Comércio e dos Serviços Sustentáveis, Departamento de Silvicultura, Viveiro da Floresta, Rodovia AC 40, Km 03, 1377, Loteamento Santa Helena, CEP 69908-642, Rio Branco (AC), Brasil. andre.schatz@ac.gov.br 
madeireiros mais conhecidos da Amazônia e, com grande potencial de promover a valorização e o uso econômico da floresta (GOMES, 2010). Este produto tem demanda internacional e é utilizado para iluminação, confecção de sabão e velas, na indústria de cosméticos, como repelente de insetos e na medicina popular (FAZOLIN et al., 2000; FERRAZ et al., 2002; SHANLEY e LONDRES, 2010; MELO et al., 2011).

Em área de terra firme, as sementes são encontradas sob as copas das árvoresmatrizes (McHARGUE e HARTSHORN, 1983) e são coletadas soltas ou dentro dos frutos, de preferência, logo após a queda destes (FERRAZ et al., 2002), pois são rapidamente dispersas e consumidas por catetos, queixadas, cotias, pacas e/ ou atacadas por insetos, especialmente os do gênero Hypsipyla (McHARGUE e HARTSHORN, 1983; GUARIGUATA et al., 2000; PLOWDEN, 2004).

Sementes de andiroba broqueadas foram encaminhadas até o Laboratório de Entomologia da Embrapa Acre, a fim de se determinar o agente causador dos danos. As sementes foram coletadas no mês de junho de 2013, provenientes de 10 andirobeiras de ocorrência natural em terra firme e com altura aproximada de $12 \mathrm{~m}$. As árvores estavam localizadas na bordadura de um remanescente florestal, na propriedade Bom Sucesso, localizada no município de Senador Guiomard - AC (6748'26.35's; $\left.10^{\circ} 06^{\prime} 31.67^{\prime \prime} \mathrm{W}\right)$.

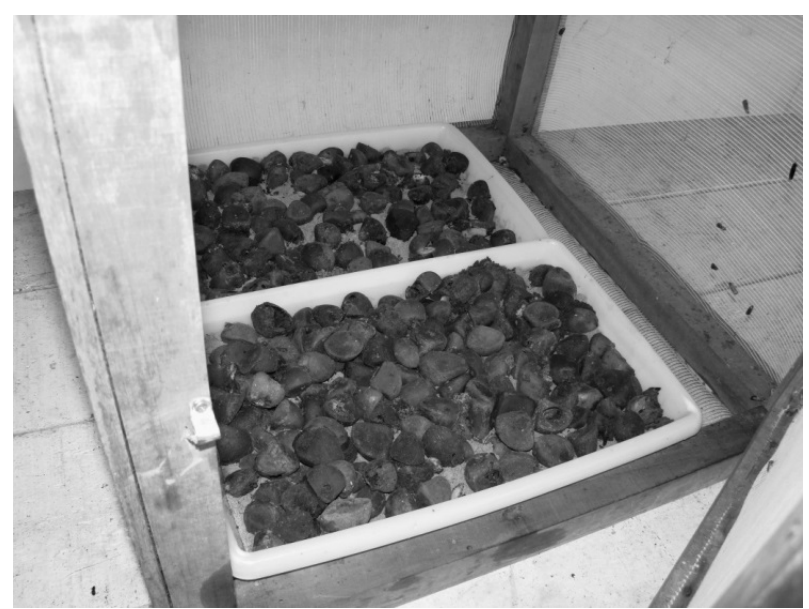

FIGURA 1: Sementes de andiroba dispostas em bandejas plásticas contendo areia umedecida e acondicionadas no interior de gaiola telada.

FIGURE 1: Andiroba seeds deposited in plastic trays containing moistened sand and placed into a net cage.
Foi examinado um total de 240 sementes, as quais foram divididas em dois lotes, com igual número de sementes, dispostas em bandejas plásticas contendo areia umedecida e acondicionadas no interior de uma gaiola telada, com dimensões de 60 x 60 x $60 \mathrm{~cm}$ (Figura 1).

Diariamente, a gaiola foi vistoriada à procura de insetos emergidos, durante um período de 30 dias. Neste período foram capturados 36 lepidópteros adultos, os quais foram montados em alfinetes entomológicos, preservados em via seca e enviados para identificação taxonômica. Também foram observadas moscas da família Stratiomyidae (Diptera), comumente associadas à matéria orgânica em decomposição (LARDÉ, 1990).

Os lepidópteros foram identificados como Hypsipyla ferrealis Hampson (Lepidoptera: Pyralidae) (Figura 2), pelo taxonomista Dr. Vítor Osmar Becker (Instituto Uiraçu, Camacan - BA), sendo alguns exemplares depositados na Coleção Entomológica da Embrapa Acre, Rio Branco - AC.

Duas espécies de Hypsipyla são relatadas associadas à Carapa spp. no Brasil: Hypsipyla ferrealis, conhecida como broca-das-sementes da andiroba e Hypsipyla grandella Zeller (Lepidoptera: Pyralidae), conhecida como broca-dos-ponteiros, que pode atacar tanto os frutos, quanto os ramos (gemas terminais) e, cujo ataque é caracterizado pela exsudação de goma e serragem nos brotos (GALLO et al., 2002).

A predação de sementes por insetos implica em danos diretos na qualidade das sementes e pode alterar a regeneração das espécies, visto que as sementes predadas têm seu potencial de germinação reduzido (CRAWLEY e GILLMAN, 1989; PINTO, 2007). Além disso, a predação de sementes pode comprometer diretamente a produção de óleo, em função das perdas quantitativas de sementes e, indiretamente, diminuindo a qualidade do óleo produzido (LIMA, 2010).

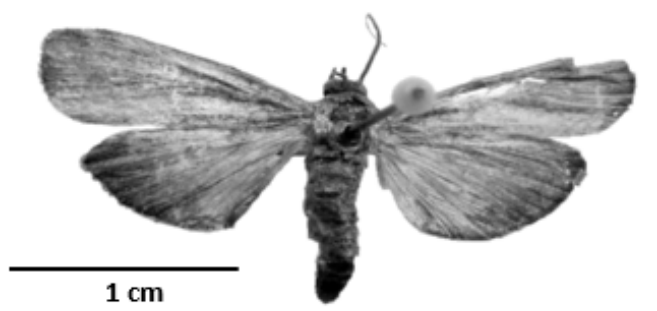

FIGURA 2: Vista dorsal de adulto de Hypsipyla ferrealis.

FIGURE 2: Dorsal view of an adult Hypsipyla ferrealis. 
As espécies Hypsipyla ferrealis e Hypsipyla grandella são consideradas os insetos-pragas mais importantes que atacam espécies arbóreas da família Meliaceae, tais como o mogno, o cedro e a andiroba, atacando tanto as sementes como a própria planta (LIMA, 2010). As larvas de ambas as espécies alimentam-se dos cotilédones das sementes (FERRAZ et al., 2002; QUERINO et al., 2008) (Figura 3), diminuindo sua capacidade germinativa ou inviabilizando as mesmas. Guedes et al. (2008) verificaram uma perda de $42 \%$ das sementes de andiroba, predadas por Hypsipyla, em área de várzea no Amapá e Mellinger (2006), em torno de $25 \%$ de sementes, no Amazonas.

De acordo com Querino et al. (2008), o ataque por Hypsipyla grandella em andirobeiras, pode retardar o desenvolvimento e até mesmo levar à morte da planta. Em contrapartida, Hypsipyla ferrealis, que ataca principalmente as sementes, prejudica a reprodução da espécie, bem como a produção de óleo. Desta forma, o ataque desses lepidópteros causa enormes perdas econômicas, restringindo o estabelecimento e cultivo de muitas espécies de meliáceas (FERRAZ et al., 2003).

Em face do mercado promissor e em expansão, Menezes (2005) recomenda que sejam realizadas pesquisas, visando determinar métodos de controle eficientes, em decorrência do ataque de pragas nas sementes de andiroba, a fim de reduzir as perdas na produção (VIEIRA et al., 2001; GUEDES et al., 2008).

Jordão e Silva (2006) sugerem que vários métodos de controles sejam combinados para minimizar a infestação de Hypsipyla ferrealis, haja vista a dificuldade de se conseguir resultados eficazes. Ferraz et al. (2003) recomendam, como forma de minimizar a perda, imergir as sementes

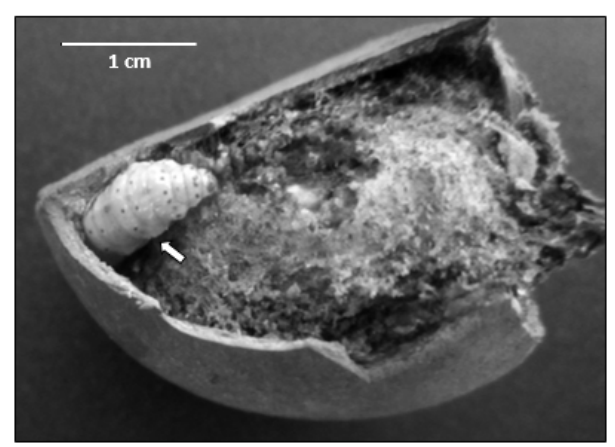

FIGURA 3: Aspecto interno de fruto de andiroba predado por Hypsipyla ferrealis.

FIGURE 3: Inside aspect of Andiroba fruit preyed by Hypsipyla ferrealis. em água, por pelo menos 24 horas, para eliminar as larvas por afogamento.

De acordo com sua distribuição geográfica na Amazônia brasileira, Hypsipyla ferrealis já foi relatada infestando sementes de Carapa guianensis nos estados do Pará, Roraima, Amapá e Amazonas (BECKER, 1971; JORDÃO e SILVA, 2006; PINTO, 2007; QUERINO et al., 2008). Portanto, o registro desta espécie atacando sementes de Carapa guianensis no Estado do Acre, contribui para aumentar o conhecimento de sua distribuição geográfica na região amazônica, bem como no Brasil.

\section{CONCLUSÕES}

Este é o primeiro registro de Hypsipyla ferrealis no Estado do Acre, infestando sementes de Carapa guianensis.

\section{AGRADECIMENTOS}

Ao taxonomista DSc. Vítor Osmar Becker (Instituto Uiraçu, Camacan - BA) pela identificação de Hypsipyla ferrealis e à Ma. Luciana Maira de Sales Pereira (Instituto Federal do Acre - IFAC), pela revisão do Abstract e texto.

\section{REFERÊNCIAS BIBLIOGRÁFICAS}

BECKER, V.O. Microlepidópteros que vivem nas essências florestais do Brasil. I. Hypsipyla ferrealis (Hampson) (Lepidoptera, Pyralidae, Phycitinae), broca da semente da andiroba - Carapa guianensis Aubl. (Meliaceae). Revista Floresta, v. 3, p. 85-90, 1971.

BOUFLEUER, N.T. Aspectos ecológicos de andiroba (Carapa guianensis Aubl., Meliaceae), como subsídios ao manejo e conservação. 2004. 84f. Dissertação de Mestrado em Ecologia e Manejo de Recursos Naturais - Universidade Federal do Acre, Rio Branco, AC.

CRAWLEY, M.J.; GILLMAN, M.P. Populationdynamics of cinnabar moth and ragwort in Grassland. Journal of Animal Ecology, v. 58, p. 1035-1050, 1989.

FAZOLIN, M.; ESTRELA, J.L.V.; PESSOA, J.S. Avaliação do uso do óleo de andiroba Carapa guianensis Aubl., no controle da Cerotoma tingomarianus Bechyné em feijoeiro no Acre. 2000. In: CONGRESSO BRASILEIRO DE DEFENSIVOS AGRÍCOLAS NATURAIS, 
Fortaleza. Anais... Fortaleza: Academia Paraense de Ciências.

FERRAZ, I.D.K.; CAMARGO, J.L.C.; SAMPAIO, P.T.B. Andiroba: Carapa guianensis Aubl. \& Carapa procera D.C., Meliaceae. INPA: Manual de Sementes da Amazônia, Fascículo 1., 2003. 4p.

FERRAZ, I.D.K.; CAMARGO, J.L.C.; SAMPAIO, P.T.B. Sementes e plântulas de andiroba (Carapa guianensis Aubl. e Carapa procera D.C.): Aspectos botânicos, ecológicos e tecnológicos. Acta amazônica, v. 32, n. 4, p. 647-661, 2002.

GALLO, D. et al. Entomologia agrícola. Piracicaba: Fealq., 2002. 920p.

GOMES, H.S.R. Estrutura populacional e produção de andiroba em terra firme e várzea no sul do Amapá. 2010. 82f. Dissertação de Mestrado em Biodiversidade Tropical - Universidade Federal do Amapá, Macapá, AP.

GUARIGUATA, M.R.; ADAME, J.J.R.; FINEGAN, B. Seed removal and fate in two selectively logged lowland forests with contrasting protection levels. Conservation Biology, v. 14, n. 4, p. 1046-1054, 2000.

GUEDES, M.C.; SOUTO, E.B.; CORREA, C.; GOMES, H.S.R. Produção de sementes e óleo de andiroba em área de várzea do Amapá. 2008. In: SEMINÁRIO DE MANEJO SUSTENTÁVEL DE PRODUTOSFLORESTAIS NÃO-MADEIREIROS NAAMAZÔNIA, Rio Branco, Anais... Rio Branco, p. 111-120.

JORDÃO, A.L.; SILVA, R.A. Guia de pragas agrícolas para o manejo integrado no Estado do Amapá. Holos: Ribeirão Preto., 2006. 182p.

LARDÉ, G. Recycling of coffee pulp by Hermetia illucens (Diptera: Stratiomyidae) larvae. Biological Wastes, v. 33, p. 307-310, 1990.

LIMA, A.S. Produção, biometria e germinação de andirobeiras (Carapa spp.) da APA da Fazendinha, Macapá - AP. 2010. 52f. Trabalho de Conclusão de Curso em Manejo Florestal - Universidade do Estado do Amapá (UEAP), Macapá.

MELLINGER, L.L. Aspectos da regeneração natural e produção de sementes de Carapa guianensis Aubl. (andiroba), na reserva de desenvolvimento sustentável Amanã, AM. 2006. 81f. Dissertação de Mestrado em Ciências Biológicas - Universidade Federal do Amazonas, INPA/UFAM, Manaus.

MELO, M.S.; ALMEIDA, E.C.; DANTAS, J.B. Boas práticas de manejo e extração de óleo vegetal de andiroba. Santarém: IBAMA, 2011. $71 \mathrm{p}$.

MENDONÇA, A.P.; FERRAZ, I.D.K. Óleo de andiroba: processo tradicional da extração, uso e aspectos sociais no estado do Amazonas, Brasil. Acta Amazonica, v. 37, n. 3, p. 353-364, 2007.

MENEZES, A.J.E. O histórico do sistema extrativo e a extração de óleo de andiroba cultivado no município de Tomé-Açu, estado do Pará. 2005. In: CONGRESSO DA SOBER, 42. Anais... Sociedade Brasileira de Economia e Sociologia Rural, p. 2-11. McHARGUE, L.A.; HARTSHORN, G.S. Seed and seedling ecology of Carapa guianensis. Turrialba, v. 33, n. 4, p. 399-404, 1983.

PINTO, A.A. Avaliação de danos causados por insetos em sementes de andiroba (Carapa guianensis Aubl.) e andirobinha ( $C$. procera) (Meliaceae) na reserva florestal Ducke em Manaus, AM. Brasil. 2007. 80f. Dissertação de Mestrado em Ciências Biológicas - Instituto Nacional de Pesquisa da Amazônia, Universidade Federal do Amazonas, Manaus, AM.

PLOWDEN, C. The ecology and harvest of andiroba seeds for oil production in the Brazilian Amazon. Conservation \& Society, v. 2, n. 2, p. 251-270, 2004.

QUERINO, R.B.; MARSARO JÚNIOR, A.L.; TELES, A.S.; COSTA, J. de A.M. Predação de sementes de andiroba (Carapa spp.) por Hypsipyla ferrealis Hampson (Lepidoptera: Pyralidae) em Roraima. Embrapa: Boletim de Pesquisa e Desenvolvimento, Boa Vista, n. 5, p. 19, 2008.

SHANLEY, P.; LONDRES, M. Andiroba. In: SHANLEY, P.; SERRA, M.; MEDINA, G. Frutíferas e plantas úteis na vida amazônica. $2^{\mathrm{a}}$ ed., Brasília: Cifor., 2010. 316p.

VIEIRA, A.H. et al. Técnicas de produção de sementes florestais. (Embrapa Rondônia, Circular técnica, 205), 2001. 4p. 